\title{
Dosimétrie individuelle, grandeurs et unités
}

\author{
A. BIAU
}

Dès 1928, à la création de la Commission internationale de protection radiologique $(\mathrm{CIPR})^{1}$ des recommandations sont produites en fixant des limites d'exposition pour éviter les risques déterministes. Les risques déterministes considérés étaient les érythèmes observés dans les pratiques de radiologie médicale. Ainsi la première limite de 1934 correspondait au dixième de la dose provoquant un érythème (voir chapitre 4). Pour se situer par rapport à ces limites il fallait bien apprécier le niveau d'exposition par calcul ou par mesure.

$\mathrm{Au}$ fil du temps, les mesures seront effectuées à partir de radiamètres (compteurs Geiger Muller puis chambre d'ionisation), de détecteurs d'ambiance et de dosimètres individuels photographiques et stylos dosimètres (condensateurs se déchargeant sous l'effet des rayonnements).

\subsection{L'exposition et le Roentgen}

Pour la mesure sur l'individu la première grandeur mesurée a été l'exposition, grandeur liée au faisceau de rayonnements incidents, photons $\gamma$ ou X et dont l'unité était le Roentgen (R). Durant longtemps les radiamètres ont été gradués en Roentgen et il doit y en avoir encore!

Le Roentgen est défini comme le quotient de la somme des charges électriques de tous les ions du même signe produits par l'ionisation de l'air dans un certain volume par la masse de ce volume d'air :

$$
1 \text { Roentgen }=2,58.10^{-4} \text { Coulomb } / \mathrm{kg}
$$

Jusque dans les années 1970 les résultats de certains laboratoires ont été exprimés en Roentgen.

\footnotetext{
1 En anglais ICRP. À l'origine, en 1928, a été crée l'ICRPX, International X-ray and Radium Protection Commission à l'occasion du Deuxième Congrès International de Radiologie à Stockholm (Suède). En 1950, cette commission a pris le nom actuel (ICRP) pour tenir compte de la rapide évolution du champ d'application de la radioprotection en dehors du domaine médical.
} 


\subsection{La dose absorbée et le rad}

Par la suite, la grandeur dosimétrique de base en radioprotection a été la dose absorbée, grandeur représentative de l'action des rayonnements ionisants et définie comme le quotient de l'énergie des rayonnements ionisants, quelle que soit leur nature, absorbée dans un élément de volume de matière divisée par la masse de ce volume.

L'unité de dose absorbée est le rad (radiation absorbed dose) :

$$
1 \mathrm{rad}=100 \mathrm{ergs} / \mathrm{g}=10^{-2} \text { joule } / \mathrm{kg}
$$

On a souvent utilisé pour les photons indifféremment le roentgen ou le rad car l'exposition en roentgen et la dose absorbée en rad étaient numériquement très proches. En effet la relation entre ces deux grandeurs est, pour les photons :

$$
\text { Dose dans l'air (rad) } \quad \mathrm{D}_{\mathrm{a}}=0,87 \mathrm{R}
$$

Dose dans le tissus humain (rad) $\mathrm{D}_{\mathrm{T}}=0,94 \mathrm{R}$

Compte tenu des incertitudes sur les mesures de radioprotection la confusion de ces deux unités n'entrainait pas une erreur bien importante et allait dans le sens de la protection lorsque la valeur numérique de l'exposition était utilisée pour exprimer la dose absorbée en rad.

\subsection{L'équivalent de dose et le rem}

L'équivalent de dose est la dose absorbée pondérée par un facteur de qualité lié à la nature du rayonnement, égal à 1 pour les photons et les électrons, 10 pour les neutrons. Ce facteur de qualité $\mathrm{Q}$ est défini, à partir des Recommandations de la CIPR, publication $n^{\circ} 2$ de 1958 (ICRP, 1959), dans le décret du 20 juin 1966 selon les termes suivants : «Facteur de qualité : fonction du transfert linéique d'énergie pour pondérer les doses absorbées afin de rendre compte de leur signification pour les besoins de la radioprotection ».

Les valeurs de ce facteur Q figurant dans le tableau I resteront identiques jusqu'à la mise en application du décret du 31 mars 2003.

L'unité d'équivalent de dose est le rem (rad equivalent man).

Jusqu'à la fin des années 1970 les résultats de dosimétrie individuelle ont été exprimés en rems ou plutôt en millirems (millièmes de rems), les mesures étant effectuées sur un dosimètre porté au niveau de la poitrine pour évaluer l'exposition globale de l'organisme. Lorsque le risque d'exposition est localisé au niveau des mains, un ou deux dosimètres complémentaires peuvent être portés au poignet. 
Au début des années 1980 pour des raisons d'application du Système International d'Unités de nouvelles unités ont été définies pour la dose absorbée et l'équivalent de dose absorbée.

\subsection{La dose absorbée et le Gray (Gy)}

La dose absorbée garde sa définition initiale mais est désormais exprimée en Gray correspondant à une énergie de 1 joule absorbée dans 1 kilogramme de matière :

$$
1 \mathrm{Gray}=1 \text { joule } / \mathrm{kg} \text { et } 1 \mathrm{Gray}=100 \mathrm{rads}
$$

\subsection{L'équivalent de dose et le Sievert (Sv)}

L'équivalent de dose est défini comme le produit de la dose absorbée par le facteur de qualité correspondant au type de rayonnement en cause. Il est exprimé en Sievert (Sv) :

$$
\begin{gathered}
1 \text { Sievert }=1 \text { Gray } \times Q \text { et } 1 \text { Sievert }=100 \text { rems } \\
Q=\text { facteur de qualité }
\end{gathered}
$$

Le facteur de qualité $\mathrm{Q}$ (Tab. I) dépend de la nature des rayonnements et de leur transfert linéique d'énergie (TLE) correspondant à l'énergie transférée à la matière irradiée par unité de longueur en $\mathrm{keV} / \mu \mathrm{m}$.

\section{TABLEAU I}

Facteurs de qualité des rayonnements.

\begin{tabular}{lc}
\hline Rayonnements & $\mathrm{Q}$ \\
\hline Rayonnements X, $\gamma$, électrons et positrons & 1 \\
Neutrons et protons & 10 \\
Particules $\alpha$ & 20 \\
\hline
\end{tabular}

Enfin la CIPR dans sa publication n ${ }^{\circ} 60$, parue en 1991 (ICRP, 1991) a émis des nouvelles recommandations après celles de 1958 et défini deux nouvelles grandeurs dosimétriques de base en radioprotection, la dose équivalente et la dose efficace.

\subsection{La dose équivalente et le Sievert}

La dose équivalente $\mathrm{H}_{\mathrm{T}}$ est la dose absorbée $\mathrm{D}_{\mathrm{R}}$ à un organe ou un tissu T, pondérée par un facteur lié à la qualité et le type de rayonnement, $\mathbf{w}_{\mathbf{R}}$ (voir Tab. II)

$$
\mathbf{H}_{\mathbf{T}}=\mathbf{w}_{\mathbf{R}} \cdot \mathbf{D}_{\mathbf{R}}
$$

La dose équivalente s'exprime en Sievert. 
Tableau II

Facteurs de pondération radiologique.

\begin{tabular}{lc}
\hline Nature et énergie (E) des rayonnements & Facteur de pondération radiologique $\mathrm{W}_{\mathrm{R}}$ \\
\hline Photons toutes énergies & 1 \\
Électrons, muons (toutes énergies) & 1 \\
Neutrons $\quad \mathrm{E}<10 \mathrm{keV}$ & 5 \\
$10 \mathrm{keV}<\mathrm{E}<100 \mathrm{keV}$ & 10 \\
$100 \mathrm{keV}<\mathrm{E}<2 \mathrm{MeV}$ & 20 \\
$2 \mathrm{MeV}<\mathrm{E}<20 \mathrm{MeV}$ & 10 \\
$\mathrm{E}>20 \mathrm{MeV}$ & 5 \\
Protons $\quad \mathrm{E}>2 \mathrm{MeV}$ & 5 \\
Particules $\alpha$, fragments de fissions, Noyaux lourds & 20 \\
\hline
\end{tabular}

\subsection{La dose efficace et le Sievert}

La dose efficace E est égale à la somme des doses équivalentes pondérées par un facteur lié à la sensibilité aux rayonnements des organes ou tissus concernés, $\mathbf{w}_{\mathbf{T}}$ (voir Tab. III)

$$
\mathbf{E}=\Sigma_{\mathbf{T}} \mathbf{w}_{\mathbf{T}} \Sigma_{\mathbf{R}} \mathbf{w}_{\mathbf{R}} \mathbf{D}_{\mathbf{T R}}
$$

Tableau III

Facteurs de pondération tissulaire (CIPR 60 et décret du 31 mars 2003) ${ }^{2}$.

\begin{tabular}{lc}
\hline Tissu ou organe & Facteur de pondération tissulaire $\mathrm{W}_{\mathrm{T}}$ \\
\hline Gonades & 0.20 \\
Moëlle osseuse & 0.12 \\
Colon & 0.12 \\
Poumons & 0.12 \\
Estomac & 0.12 \\
Vessie & 0.05 \\
Seins & 0.05 \\
Foie & 0.05 \\
Esophage & 0.05 \\
Thyrö̈de & 0.05 \\
Peau & 0.01 \\
Surface des os & 0.01 \\
Autres tissus ou organes & 0.05 \\
\hline
\end{tabular}

2 La publication 103 de la CIPR parue en 2007 prévoit quelques modifications de ces valeurs pour tenir compte de recherches récentes. 
La somme des $\mathbf{w}_{\mathbf{T}}$ est égale à 1 de sorte que dans le cas où un individu est soumis à une exposition globale et homogène de son corps, la dose efficace est numériquement égale à la dose équivalente corps entier.

\subsection{Grandeurs dosimétriques opérationnelles}

Les grandeurs de radioprotection $\mathrm{E}$ et $\mathrm{H}_{\mathrm{T}}$ définies ci-dessus ne sont pas directement mesurables, c'est pourquoi pour les évaluer le plus précisément possible, ont été définies des grandeurs dosimétriques opérationnelles dont deux sont plus particulièrement adaptées à l'utilisation des dosimètres individuels, il s'agit de :

- $\quad H_{p}(10)=$ équivalent de dose individuel à $10 \mathrm{~mm}$ de profondeur dans les tissus

\section{- $\quad H_{p}(0.07)=$ équivalent de dose individuel à $0.07 \mathrm{~mm}$ ou $70 \mu \mathrm{m}$}

Deux autres grandeurs opérationnelles, l'équivalent de dose ambiant $\mathrm{H}^{*}(\mathrm{~d})$ et l'Equivalent de dose directionnel $\mathrm{H}^{\prime}(\mathrm{d}, \Omega)$ ont été définies pour caractériser les conditions d'exposition à partir de mesures dans une sphère de référence définie par l'ICRU. Les mesures correspondantes se font localement par des PCR qui n'ont pas nécessairement recours aux laboratoires de dosimétrie passive.

Concrètement $\mathrm{H}_{\mathrm{p}}(10)$ est mesuré sous écran équivalent à $10 \mathrm{~mm}$ de tissu et représente la dose équivalente en profondeur et $\mathrm{H}_{\mathrm{p}}(0.07)$ est mesuré sur une zone découverte du dosimètre et indique la dose équivalente à la peau.

Dans la pratique courante la valeur de $H_{P}(10)$ mesurée sur le dosimètre porté au niveau de la poitrine est considérée comme la valeur de la dose efficace et $H_{p}(0.07)$ la dose équivalente à la peau.

Tous ces nouveaux concepts initiés par la ICRP dans sa publication 60, définis dans les publications 74 de la ICRP (ICRP, 1996) et 51 de l'ICRU (ICRU, 1993), ont été repris dans la Directive 96/29 du 13 mai 1996 (EURATOM, 1996), transcrite en droit français dans le décret du 31 mars 2003 (voir chapitre 4).

Les dosimètres, quels qu'ils soient, donnent une mesure de la dose à laquelle ils sont soumis en fonction des conditions d'exposition, de la nature et de l'énergie des rayonnements. Pour mesurer la dose reçue par un individu il faut se référer à des mesures sur un ou plusieurs dosimètres portés par cet individu.

La figure ci-dessous, extraite d'un manuel de radioprotection de 1969 destiné aux manipulateurs de radiologie médicale, montre qu'il n'est pas réaliste de porter une multitude de dosimètres. 


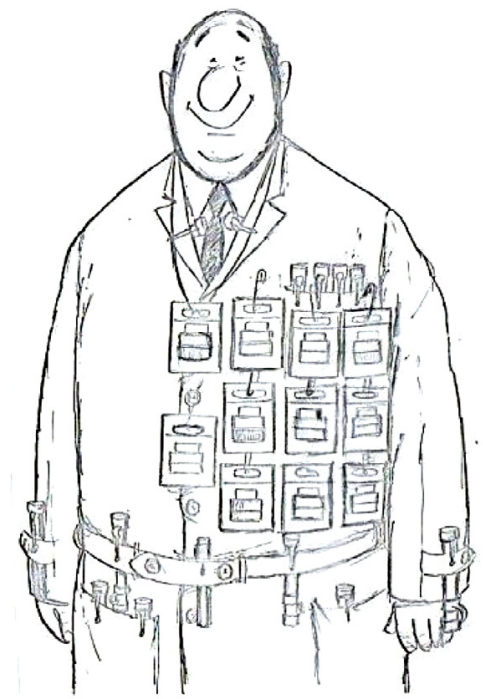

Figure 1.1 - Comment porter le dosimètre !

En routine dans le cas le plus général où le champ de rayonnement est considéré comme homogène, la mesure sur un seul dosimètre porté au niveau de la poitrine est présumée représenter la dose corporelle totale et la dose efficace.

Il est évident que c'est une simplification pratique car en réalité cette égalité ne vaut que si le porteur du dosimètre est exposé de façon complètement homogène sur l'ensemble du corps, ce qui n'est pratiquement jamais le cas.

Les incertitudes dues à la mesure et la représentativité de la mesure par rapport à la dose efficace réelle seront examinées au chapitre 3 .

Ce dosimètre de poitrine, sensé représenter la dose efficace, il faut bien sûr le porter sous les équipements de protection individuelle telles que le tablier de plomb en radiologie médicale, c'est un sujet de discussion avec les utilisateurs qui nous disent : » si on met le dosimètre sous le tablier de plomb, il n'y a pas de dose »

C'est bien là l'intérêt du tablier plombé ! Un dosimètre de complément, par exemple en bordure du tablier à l'extérieur proche de la thyroïde, peut être utile. 
Lorsqu'il y a un risque d'exposition au niveau des mains, il est utile de porter un dosimètre complémentaire au poignet et dans certains cas, de dosimètres spécialisés sous forme de bagues pour déterminer l'exposition des doigts.

\section{RÉFÉRENCES}

EURATOM (1996) Directive EURATOM n 96/29 du 13 mai 1996.

ICRP Publication 2 (1959) Recommendations of the International Commission on Radiological Protection, Pergamon Press.

ICRP Publication 60 (1991) Recommendations of the International Commission on Radiological Protection, Pergamon Press.

ICRP Publication 74 (1996) Conversion Coefficinets for use in Radiological Protection against External Radiation, Ann. ICRP 26, $\mathrm{n}^{\circ}$ 3/4.

ICRU Report 51 (1993) Quantities and Units in Radiation Protection Dosimetry (Dosimetry and measurements). 